\title{
Analysis of rotating object using video tracker
}

\author{
Djoko Untoro Suwarno* \\ Electrical Engineering Department, Sanata Dharma University, \\ Maguwoharjo, Sleman 55284, Indonesia
}

\begin{abstract}
The purpose of this paper is to show the use of video tracker to analyze circular motion. Measurement of circular motion such as angular velocity and angular acceleration is not easy to do observation and measurement directly. Through the video tracker tool, the analysis of speed and rotation acceleration becomes easier. The rotating object used as a research object is a spinner fidget. The initial velocity of the rotation depends on the pull of the finger on the spinner. The duration of rotating spinner fidget depends on the inertia and attenuation that occurs. Camera position and resolution of the camera greatly determine the data on the video tracker. The standard camera capability of 30 frames per second is less capable of capturing objects that rotate with high resolution.
\end{abstract}

Keywords: angular acceleration, angular velocity, fidget spinner, rotating object, video tracker

Received: August 7, 2017; Accepted: September 14, 2017; Published online: November 1, 2017

*Corresponding author: joko_unt@usd.ac.id

Citation: Suwarno, D. U. (2017). Analysis of rotating object using video tracker. Journal of Science and Science Education, 1(2), 75-80.

\section{Introduction}

One of the newest toy trends today is the fidget spinner. The fidget spinner consists of a ball bearing located center on a metal body. The most common fidget spinner consists of three lobes. This toy is held in the middle and played with fingers. Players rotate the fidget spinner and wait until the fidget spinner stops spinning. The spin time of this fidget spinner depends on the initial rotation speed, inertia, and deceleration. Many rotating objects are found around us, such as spinning motors. Measuring characteristics of rotating objects is not easily observable and measured directly. Measurements of rotating objects include acceleration and deceleration of the rotation of objects. Rhett $(2017 \mathrm{a}, \mathrm{b})$ estimated the spin time by using the FFT methods. Rhatt also estimates the moment of inertia of the fidget spinner to estimate spin time.

In this paper, rotating objects measured using video tracker software. Through the video tracker software can be shown the acceleration and deceleration of rotating objects. Video Tracker is software that is open source and developed by Douglas Brown. The video tracker can be downloaded freely at www.opensourcephysics.org. A video tracker is a tool for analyzing the motion of a video. (Suwarno, 2015) conducts a pendulum vibration analysis using a video tracker and can show an underdamped oscillation motion until over damped oscillation.

\section{Materials and Methods}

The research was conducted using rotary objects; fidget spinner, cameras, video tracker software and DIY frequency counter (period measurement). The fidget spinner is rotated using finger. Rotating objects were recorded using the camera and analyzed using the video tracker. The rotating part of 
the object is marked track for each frame. The data obtained in the form of point $(x, y)$ as a function of time.

\subsection{Experiment setup}

The experiments setup is shown in Figure 1. The rotary object is recorded using a digital camera supported by a tripod. The video file is tracked using the video tracker. The things that are used as rotating objects are turntable for cake decoration and a fidget spinner.
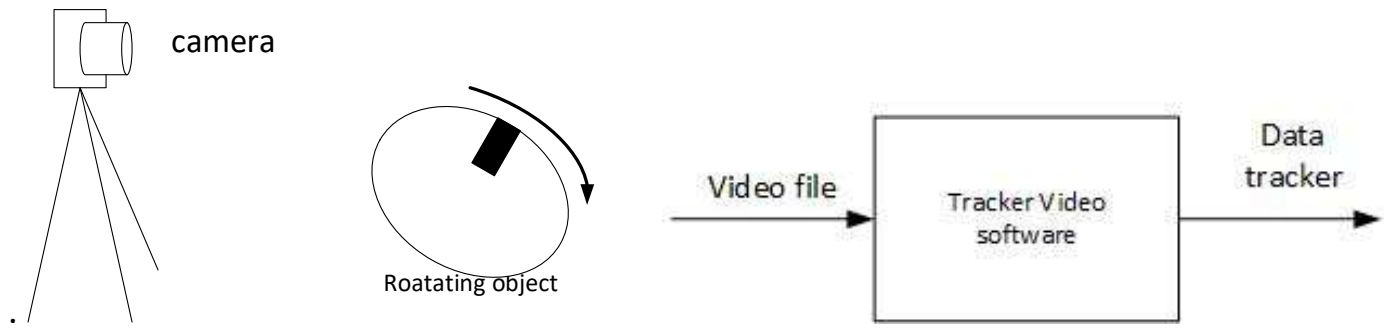

Figure 1. Experiment setup.

The equation of a rotary object (Serway et al., 2000) is shown in the Eq. (1).

$$
s=\theta \cdot R \text {, }
$$

where $s$ is circular trajectory (in $\mathrm{m}$ ), $\theta$ is angular (in radian), and $R$ is radius (in $\mathrm{m}$ ). Angular velocity $\omega$ is calculated by using Eq. (2).

$$
\omega=\frac{\Delta \theta}{\Delta t} .
$$

The relationship between rpm and angular velocity $\omega$ shown in Eq. (3).

$$
\omega=\frac{\mathrm{rpm}}{60} \cdot 2 \pi(\mathrm{rad} / \mathrm{s}) \text {. }
$$

Figure 2 shows turntable for cake decoration and fidget spinners as rotating objects
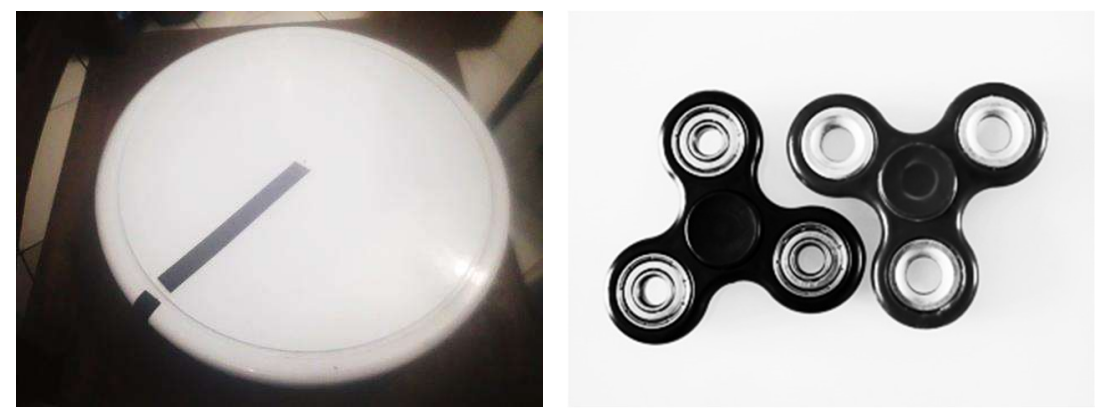

Figure 2. Turntable for cake decoration and fidget spinners.

Any spinning object has a moment of inertia. Moment inertia of the spinner fidget can be approximated by using Eq. (4).

$$
I \approx m R^{2},
$$

where $I$ is moment of inertia object (in $\mathrm{kg} \mathrm{m} 2$ ), $m$ is mass of object (in $\mathrm{kg}$ ), $R$ is radius of object rotation (in $\mathrm{m}$ ).

To rotate the spinner fidget, hold the middle section and push one of the lobes using the finger. The force applied to the spinner fidget will produce torque as shown in Eq. (5).

$$
T=F \cdot R \text {. }
$$


Force given to the spinner fidget for a certain amount of time is a change of momentum. The momentum change is written as in Eq. (6).

$$
\Delta P=F \cdot \Delta t .
$$

The momentum change in the rotating object is shown in Eq. (7).

$$
P=m \cdot \Delta v \text {. }
$$

The relationship between the velocity of $v$ and the angular velocity $\omega$ is shown in Eq. (8).

$$
v=\omega \cdot R \text {. }
$$

Eqs. (6) and (7) are substituted into Eq. (8) yields Eq. (9).

$$
\Delta \omega=\frac{F \cdot \Delta t}{m \cdot R}
$$

The change in angular velocity is the result of a mass $m$ with radius $R$ being force for a given time. The torque that occurs in a rotating object is shown in Eq. (10).

$$
\tau=I \frac{d \omega}{d t}+b \omega
$$

where $\tau$ is torque applied to object (in Nm), $I$ is moment of Inertia of rotating object (in kg m2), $\omega$ is angular velocity (in rad/s), $b$ is damping coefficient. Solution of Eq. (10) is angular velocity expressed as follows:

$$
\omega_{t}=\omega_{0} e^{-\frac{t}{T_{c}}}
$$

where $\omega_{t}$ is angular velocity at time $t$ (in rad/s), $\omega_{0}$ is initial angular velocity (in rad/s), $t$ is time (in second), $T_{C}$ is time constant defined by $T_{c}=\frac{M R^{2}}{b^{2}}$.

The fidget spinner has a mass of 60 grams and radius of $4.3 \mathrm{~cm}$. Moment of inertia of the fidget spinner is calculated as $1102 \mathrm{gr} \mathrm{cm} 2$ or $0.00011 \mathrm{~kg} \mathrm{~m} 2$. Turntable has a mass of $235 \mathrm{gram}$, with a diameter of $32.5 \mathrm{~cm}$.

\section{Results and Discussion}

The result of the video tracker is presented in Figure 3. The initial state of the object is stopped, and then the object is given force for a certain amount of time. The object accelerates for a moment, reaches its maximum speed and then decelerates until it stops. The ticker time on the video tracker depends on the frame resolution per second of the camera. For the camera with $30 \mathrm{fps}$ then there is a time interval that can be observed that is equal to $33 \mathrm{~ms}$. Data tracking lies far apart indicating greater speed compared to tracking data with close position. The tracking for the $x$-axis will form a sine function, while tracking on the $y$-axis will form a cosine function. Furthermore, Figure 4 shows the tracking data for the $x$ axis and $y$ axis will form the path of the rotating object.

Figure $5 a$ shows the angle as a function of time. The graph in Figure $5 \mathrm{a}$ is a discontinued graph. The angular unit has a 360-degree period, when angle more than 360 the angle is reduced by 360 . The $\mathrm{CW}$ rotary direction will show a negative angle, while the CCW rotate direction will indicate a positive angle. Computational calculations there are discontinuous angles that will cause spike-like results. Figure $5 \mathrm{~b}$ shows the accumulation of angle versus time. The accumulated angle produces a continuous function. The angular velocity calculation can be done by Eq. (2).

Figure 6 shows the angular velocity as a function of time. When objects are given force and spin will experience acceleration and reach maximum speed after that the object will experience slowdown and finally stop. 


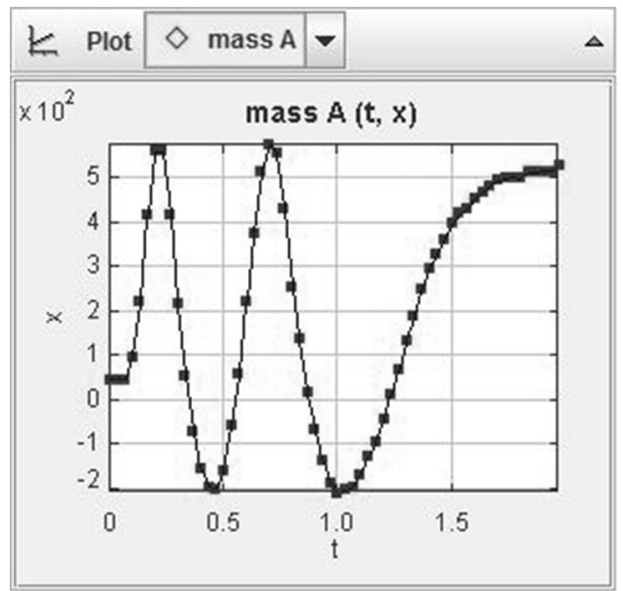

(a)

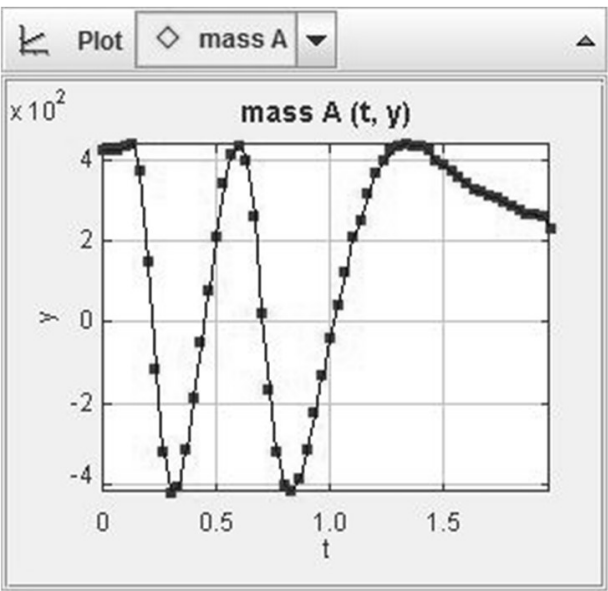

(b)

Figure 3. (a) Data tracker point $x$ versus time, (b) data tracker point $y$ vs time.

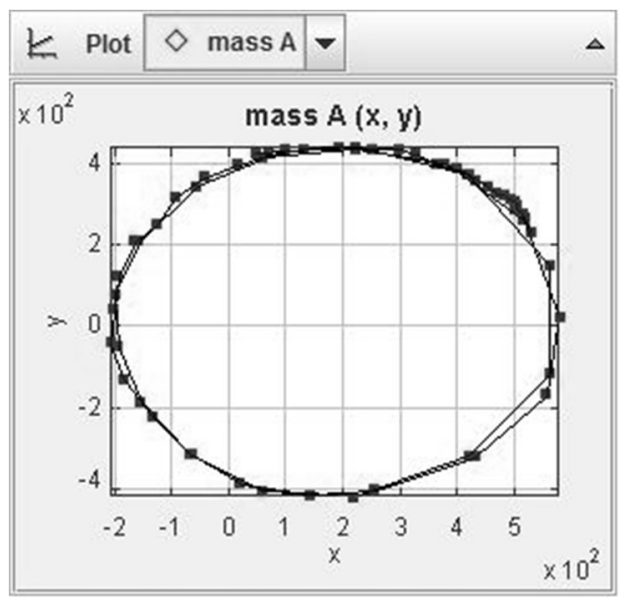

Figure 4. Data tracking for $\boldsymbol{x}$ and $y$ axis.
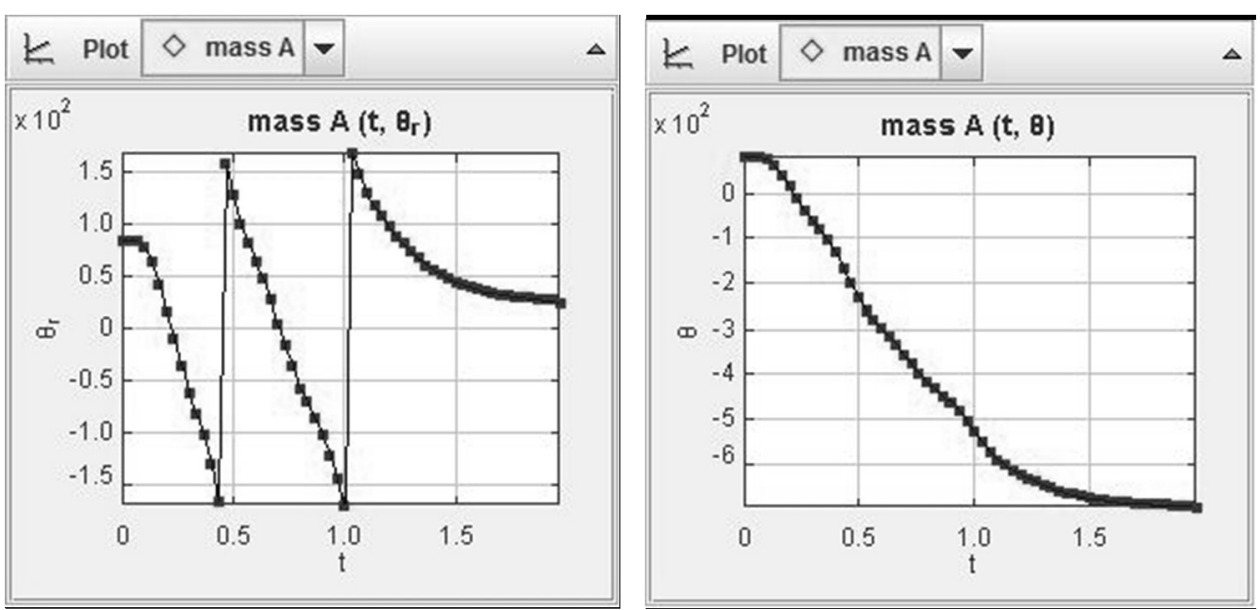

Figure 5. (a). Angle vs time, (b) acumulation angle vs time. 


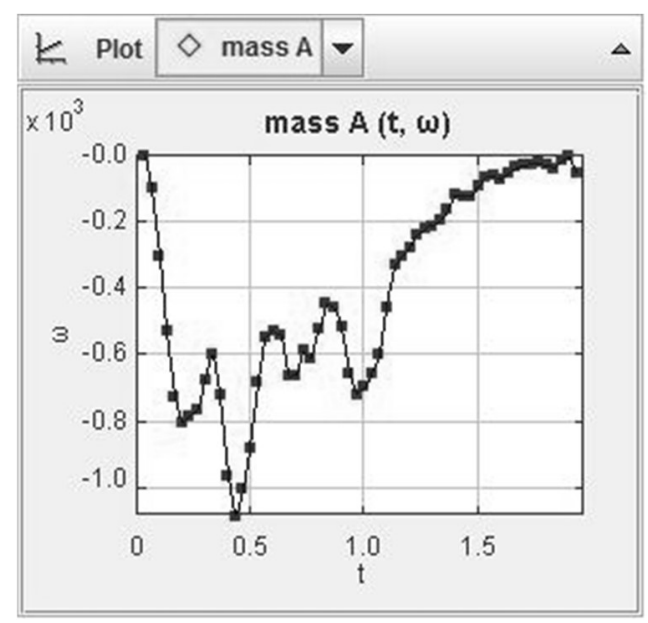

Figure 6. Angular velocity of rotating object.

Prerequisites for using video tracker:

- to get accurate results need to be done calibration using calibration stick. Without calibration, the position unit is pixel, after the calibration distance is a meter.

- to do tracking on a rotating object needs to be determined the center point so that the radius of the object does not change.

- camera position is placed perpendicular to the object to be observed. The camera position is tilted towards the object will result in tracking like an ellipse

- The observed spinning object needs to be marked so as to make tracking easier.

The advantages of using a video tracker include:

- data acquisition can be done quickly

- By doing tracking data, will be available various derived data such as speed, acceleration, angle, etc.

- Data is available in numerical form and graph

- Software is available opensource and easy to use

\section{Conclusion and Remarks}

The tracker software makes it easy to analyze rotating movements, but the rotating motions are easily observed at low rotation (<30 fps). Time to do tracking data calibration should be done first in order to get precise result. Accuracy when doing tracking data manually determines the accuracy of the results.

\section{Acknowledgment}

I thank Petrus Setyaprabowo, ST, MT for supporting to fund all the remedies for the realization of this reseach.

\section{References}

Rhett, A. (2017a). Want to know how long a fidget spinner spins? Get a laser and some physics. Retrieved from https://www.wired.com/2017/05/the-phyiscs-of-fidget-spinners/ 
Rhett, A. (2017b). Let's explore the physics of rotational motion with a fidget spinner. Retrieved from https://www.wired.com/2017/05/physics-of-a-fidget-spinner/

Serway, R. A., Beichner, R. J., \& Jewett, J. W. (2000). Physics for scientists and engineers with modern physics (5th ed.). Orlando: Saunders College Publishing.

Suwarno, D. U. (2015). Getaran osilasi teredam pada pendulum dengan magnet dan batang aluminium. Paper presented at Seminar Kontribusi Fisika 2015 